\title{
Contribution of nonconsensus base pairs within ArsR binding sequences toward ArsR-DNA binding and arsenic-mediated transcriptional induction
}

Xingjuan Chen ${ }^{1,2+}$, Xin Jiang ${ }^{4 \dagger}$, Cuijuan Tie ${ }^{4}$, Jinnon Yoo ${ }^{4}$, Yan Wang ${ }^{3}$, Meiying $X u^{1,2^{*}}$, Guoping Sun ${ }^{1,2}$, Jun Guo ${ }^{1,2}$ and Xianqiang Li $i^{1,2,4^{*}}$

\begin{abstract}
Background: A transcriptional reporter is the key component in bacterial biosensors which are employed to monitor the induction or repression of a reporter gene corresponding to environmental change. Interaction of a transcription factor with its consensus sequence generated by using a position weight matrix (PWM) model is crucial for its sensitivity of the reporter. However, recent studies suggest that PWM model based on independent contribution of individual consensus base pairs to protein interaction is often insufficient to explain complex regulation, such as the effect of nonconsensus sequences on the protein-DNA binding affinity. In the present study, we employed a simpler prokaryotic arsenic repressor (ArsR) regulation system to access the protein-DNA recognition. Contribution of nonconsensus base pairs within ArsR binding sequences toward ArsR-DNA binding and arsenic-mediated transcriptional induction was studied.

Results: We constructed a series of arsenic responsive reporters, each comprising two copies of the ArsR binding sequences from different resources. We found that high arsenic-mediated induction specifically requires the binding sequence from Escherichia coli to be placed at the first binding sequence; however, no such preference was observed for the second binding sequence, which could be from Acidithiobacillus ferrooxidans, plasmid R773, Synechococcus, or a core binding sequence of arsR. By creating a series of reporters differed at the nonconsensus base pairs of the second binding sequence, we observed that some constructs bound weakly while others strongly to ArsR. Most interestingly, although a number of these reporters showed similar binding affinity to ArsR, their arsenic-dependent induction differed significantly.

Conclusions: The results indicated that nonconsensus base pairs could have profound influence on protein binding and may also modulate post-binding function. These findings provide new insights into the complex regulation of gene expression and facilitate the development of transcriptional reporter-based biosensors.
\end{abstract}

Keywords: Bacterial biosensor, Arsenic bioreporter, Nonconsensus base pair, Arsenic repressor, Arsenic binding sequence, Protein-DNA recognition

\footnotetext{
* Correspondence: xumy@gdim.cn; jasonli@signosisinc.com

${ }^{+}$Xingjuan Chen and Xin Jiang contributed equally to this work.

'Guangdong Provincial Key Laboratory of Microbial Culture Collection and

Application, Guangdong Institute of Microbiology, Guangzhou, China

Full list of author information is available at the end of the article
}

(c) The Author(s). 2019 Open Access This article is distributed under the terms of the Creative Commons Attribution 4.0 International License (http://creativecommons.org/licenses/by/4.0/), which permits unrestricted use, distribution, and reproduction in any medium, provided you give appropriate credit to the original author(s) and the source, provide a link to the Creative Commons license, and indicate if changes were made. The Creative Commons Public Domain Dedication waiver (http://creativecommons.org/publicdomain/zero/1.0/) applies to the data made available in this article, unless otherwise stated. 


\section{Background}

The interaction between a transcription factor (TF) and its corresponding DNA binding sequence is crucial in gene regulation $[1,2]$. The base composition determines the binding affinity of the sequence to the TF. The contribution of individual base pairs in the interaction with a TF can be assessed by their conservation. Algorithms typically use the statistically simple position weight matrix (PWM) model for a binding consensus sequence $[3,4]$. Moreover, the binding consensus sequence can be determined by sequencing a group of DNA fragments or oligonucleotides selected by a TF using in vitro methods such as ChIPseq or SELEX [5]. Nevertheless, more than $40 \%$ of TFs still remain unknown for their binding sequences [5]. In Escherichia coli (E. coli), most TFs bind to a single binding site in chromosomal DNA, such as arsenic repressor (ArsR), a metalloregulatory transcriptional repressor to its operator/promoter $(\mathrm{O} / \mathrm{P})$ sequence [6]. Due to the abundant presence of ArsR binding sequences in microbial chromosomes, the alignment of these binding sequences via comparison and analysis with PWM can lead to the identification of its binding consensus sequence or motif [7]. However, recent studies suggest that PWM model based on independent contribution of individual consensus base pairs to protein interaction is often insufficient to explain various complex regulations [8], such as the effect of nonconsensus sequences on the protein-DNA binding affinity. In the present study, we employed a simpler prokaryotic ArsR regulation system to access the protein-DNA recognition.

ArsR, belonging to the Smt/ArsR family, is a regulatory protein that controls the expression of the genes involved in arsenical resistance via interaction with the arsenic-responsive operon. ArsR binding prevents the RNA polymerase from interacting with the $\mathrm{O} / \mathrm{P}$ sequence of its targeted genes in the absence of arsenicals $[7,9]$. Upon arsenic binding, the protein dissociates from the promoter, subsequently activating the gene expression [9-11]. ArsR protein is well characterized in plasmid R773 and E. coli chromosome. Both of these ArsR proteins are able to form homodimer, each with a Cys32-Val-Cys-Asp-Leu-Cys arsenic-binding sequence located at the start of their DNA binding domain [11]. ArsR from Acidithiobacillus ferrooxidans (A. ferrooxidans) does not have the binding sequence at this location, instead, their cysteine residues are located at amino acid residues of 95, 96, and 102 [12]. Both binding and the consensus sequences of Smt/ArsR family proteins, including those in $A$. ferrooxidans, have been characterized $[6,7,12-14]$.

In a previous study, we created two arsenic reporters, pLHPars9 and pLLPars9, in order to rapidly and cost- effectively monitor arsenic on site and measure arsenic bioavailability. The bioreporters pLHPars9 and pLLPars9 comprised either a high or low copy-number plasmid, along with common elements of ArsR-luciferase fusion and addition of two binding sequences, one each from $E$. coli (ECBS) and A. ferrooxidans (AFBS) chromosome, before the R773 arsR operon (arsRBC) [15]. Both of these reporters were highly sensitive to arsenite, with a low detection limit of $0.04 \mu \mathrm{M}$ arsenite $(\sim 5 \mu \mathrm{g} / \mathrm{L})$ and differed in their metal specificity, with pLLPars9 being more specific to arsenite and pLHPars9 to both arsenite and antimonite. The only difference between pLHPars9 and pLLPars 9 is their copy numbers.

In the present study, we constructed a set of arsenic bioreporters comprising two copies of different binding sequences. We found that high arsenic-mediated induction specifically requires ECBS to be placed at the first binding sequence; however, no such preference was observed for the second binding sequence. By creating a series of reporters differed at the nonconsensus base pairs of the second binding sequence, we tested the interaction of these probes with the protein. Interestingly, while some of the nonconsensus base pairs resembling the consensus are needed for the interaction with the ArsR protein, some of the nonconsensus base pairs appear to also affect the post-binding function of the TF.

\section{Results}

Arsenic transcriptional induction with a promoter containing ECBS binding sequence in arsenic bioreporters In a previous study, we found that a luciferase reporter construct pLLPars9 (or pECBS-AFBS in this study) containing ECBS-AFBS comprising two copies of ArsR binding sequences (BS), one from $E$. coli chromosome (EC) and another from A. ferrooxidans (AF) chromosomal DNA, responded better more robustly to arsenic treatment than the reporters comprising either one or two identical copies of EC or AF [15]. In this study, we swapped the position of ECBS and AFBS to create pAFBS-ECBS. After transformed into DH5a, luciferase activates of pECBS-AFBS and pAFBS-ECBS were measured and compared (Fig. 1a). Relative to untreated control cells, pAFBS-ECBS showed only 2-fold induction in arsenic-treated cells, compared to the 9-fold induction with pECBS-AFBS (Fig. 1b). This dramatic induction difference suggested that the order of these two binding sequences is crucial in the arsenic-mediated induction of the reporter.

Furthermore, we replaced the AFBS moiety within ECBS-AFBS with the binding sequence of Synechococcus smt2/1 (smt2/1BS) or arsRBC (arsRBCBS), to create the reporters pECBS-smt2/1BS and pECBS-arsRBCBS, and compared the luciferase activities of cell lysates prepared from their transformed cells with or without arsenic 

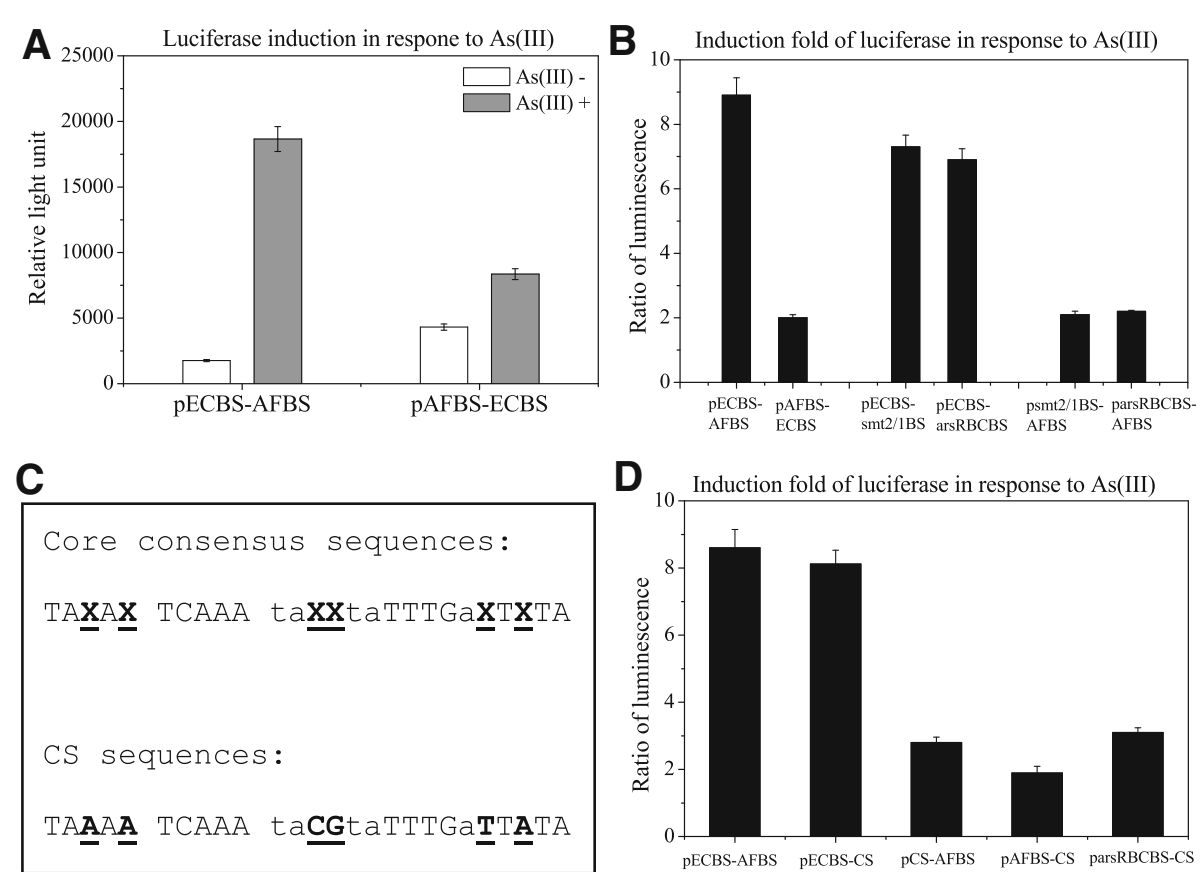

Fig. 1 Luciferase analysis of reporter constructs containing different combinations of two binding sequences. a: Luciferase activities of pECBSAFBS and pAFBS-ECBS were measured and compared between the lysates prepared from cells treated with (grey) and without (open) $10 \mu \mathrm{M}$ arsenite for $1 \mathrm{~h}$. $\mathbf{b}$ : Luciferase activities ratios of untreated to arsenic-treated cells were determined. The ratios were compared between pECBSAFBS and pAFBS-ECBS, pECBS-smt2/1BS and pECBS-arsRBCBS, and psmt2/1BS-AFBS and parsRBSBS-AFBS. C: Sequences of ArsR binding core motif and $C S$, assigned nucleotides marked with underline. D: Luciferase activities ratios of untreated to arsenic-treated cells were determined. The ratios were compared among pECBS-AFBS, pECBS-CS, pCS-AFBS, pAFBS-CS and parsRBCBS-CS

treatment. As presented in Fig. 1b, the induction of ECBSsmt2/1BS and ECBS-arsRBCBS moderately declined, losing approximately $15-25 \%$ induction folds of ECBS-AFBS. This suggested that AFBS at this position is not crucial for induction and can be substituted by other ArsR binding sequences. When we replaced ECBS moiety within ECBSAFBS with the binding sequence of smt $2 / 1$ or arsRBC to create reporters psmt2/1BS-ECBS and parsRBCBS-ECBS, we found that the ratio of luciferase activities significantly declined, losing approximately $70 \%$ compared to ECBSAFBS, as shown in Fig. 1b. The aforementioned results demonstrated that ECBS needs to be the first binding sequence in order to robustly respond to arsenic.

The consensus sequence of a DNA-binding protein can be determined by comparison of a group of binding sequences. Those consensus base pairs are believed to be crucial for the protein to bind DNA and the nonconsensus base pairs are not important to the binding. Arsenic binding proteins from different microbes are DNA-binding proteins. The DNA sequences that they bind to display a consensus sequence [7]. Our above data indicated that the second binding sequence within the biosensors was relative flexible, which could be more tolerant to bioengineering manipulation, such as a consensus sequence. According to the consensus sequence of arsRBC and cadCA, we designed a binding sequence
CS (Fig. 1c) and swapped it with the AFBS moiety to construct pECBS-CS, with 3 Ts in between. Luciferase assay revealed that pECBS-CS showed no significant difference in the response to arsenic treatment when compared to pECBS-AFBS, suggesting that the CS can be used to replace AFBS within the biosensors. However, when we swapped ECBS with the CS to make pCSAFBS, it demonstrated a significant change (Fig. 1d). Moreover, when we replaced ECBS of ECBS-CS with arsRBCBS or AFBS to construct parsRBCBS-CS and pAFBS-CS, they lost induction significantly like any other constructs without ECBS being at the first position as shown as above. These results with CS indicated that ECBS must be the first binding sequence.

\section{Arsenic cannot remove the repressor protein from AFBS- ECBS and CS-ECBS binding sequences}

To examine whether there exists any difference between ECBS-AFBS and AFBS-ECBS in ArsR binding, we performed EMSA. Biotin-labeled probes of ECBS-AFBS and AFBS-ECBS were mixed with lysates prepared from cells with and without arsenic treatment, respectively. As previous reported, two shifted bands were observed with the ECBS-AFBS probe in mock-treated cell lysate [15] and the intensity of both shifted bands dramatically declined in arsenic-treated cells (Fig. 2a), indicating that 

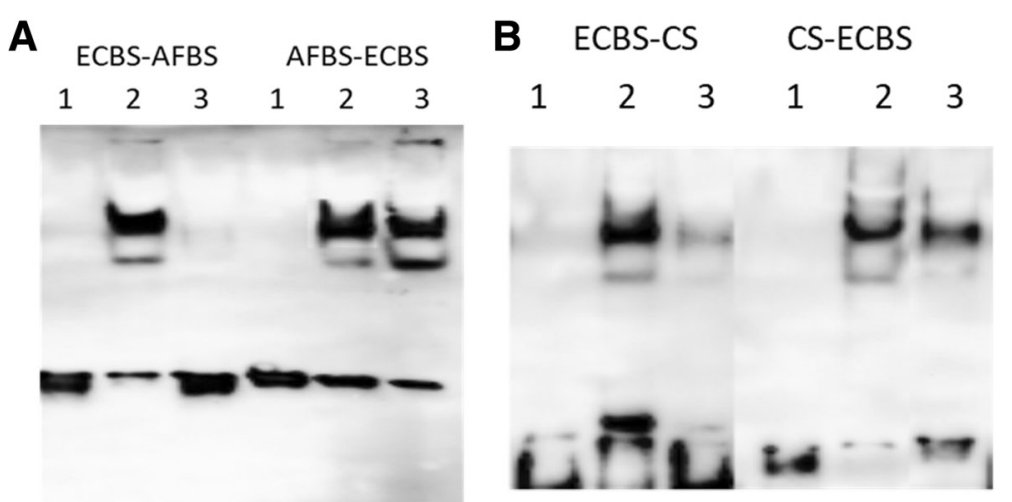

Fig. 2 Comparison of biotin-labeled probes in protein binding with EMSA. Biotin-labeled probes (1) were mixed with lysates prepared from untreated control cells (2) or arsenic treated cells (3) respectively and subjected to EMSA. a: Probe ECBS-AFBS was compared with AFBS-ECBS. b: Probe ECBS-CS was compared with CS-ECBS

the arsenic treatment disrupted the interaction between the probe and the bound repressor protein. In contrast, when we utilized the AFBS-ECBS probe to execute EMSA, we found no difference in both the number and intensity of the shifted bands between mock-treated and arsenic-treated cells. This indicated that the arsenic treatment was unable to remove the repressor protein from the AFBS-ECBS (Fig. 2a).

Next, we compared ECBS-CS and CS-ECBS probes with EMSA. The ECBS-CS probe revealed two shifted bands in control cell lysate and much weaker intensity of the shifted bands in arsenic-treated cell lysate (Fig. 2b). Again, we observed no significant difference in the intensity of the shifted bands between control and treated cells with CS-ECBS probe. This result is in accordance with AFBS-ECBS, suggesting no removal of the repressor protein from CS-ECBS probe under arsenic treatment.

\section{Arsenic removal of the repressor protein from ECBS-CS binding sequence required a linker of 3Ts}

As a linker of 3Ts was inserted between ECBS and CS, we examined whether it was necessary for the induction by removing the linker from $\mathrm{pECBS}-\mathrm{CS}$ to create pECBS-CS $(-3 \mathrm{~T})$. After transformation, the luciferase activity of pECBS-CS( $-3 \mathrm{~T})$ transformed cells were treated with and without arsenite. A significant reduction of luciferase activity in pECBS-CS transformed cells with arsenic treatment was not observed when compared to untreated control cells; however, it was observed in the pECBS-CS $(-3 \mathrm{~T})$ cells treated with arsenic (Fig. 3a; b). This result suggested that the linker of 3Ts is needed for arsenic-mediated induction.

Moreover, to examine whether the absence of 3Ts caused a steric hindrance for binding of the dimers to the binding sequences or prevented the removal of the bound repressor protein from the bound sequence, we performed EMSA with biotin-labeled probes of ECBSCS and ECBS-EC(-3Ts). As shown in Fig. 3c, like ECBS-AFBS probe, the result with ECBS-CS probe displayed two shift bands in arsenic-untreated cells but significant decline in arsenic-treated cells. Without the linker, ECBS-CS $(-3 \mathrm{~T})$ displayed two shifted bands in both control and treated cells, indicating the absence of any interference with protein binding, thus ruling out the possibility of steric hindrance. Therefore, this result suggested that the absence of the linker hampered arsenic-mediated removal of the repressor protein from the bound sequence.

\section{Fast analysis of the DNA binding sequences of ArsR with DNA filter assay}

The above results indicated that two ArsR binding elements within the biosensors were needed in order to have a sensitive response to arsenic treatment. The first element must be from E. coli and the second one was more flexible, such as arsRBCBS or CS. Although arsRBC of pECBS-arsRBCBS and CS of pECBS-CS contained the same consensus sequence, their responses to arsenic treatment were distinct with moderate difference. We assumed that the difference could arise only from the contribution of nonconsensus base pairs of the second binding sequence. To investigate the contribution of the nonconsensus base pairs in both binding and induction, we constructed a series of probes and reporters exclusively with alternative nonconsensus base pairs in the second binding site within ECBS-arsRBCBS. According to the consensus sequence of arsRBC, only 4 base pairs are not conserved. Investigation of different combinations of these 4 base pairs required testing of a large series of probes.

EMSA is usually used for monitoring protein/DNA interactions. Due to the low throughput nature of the 

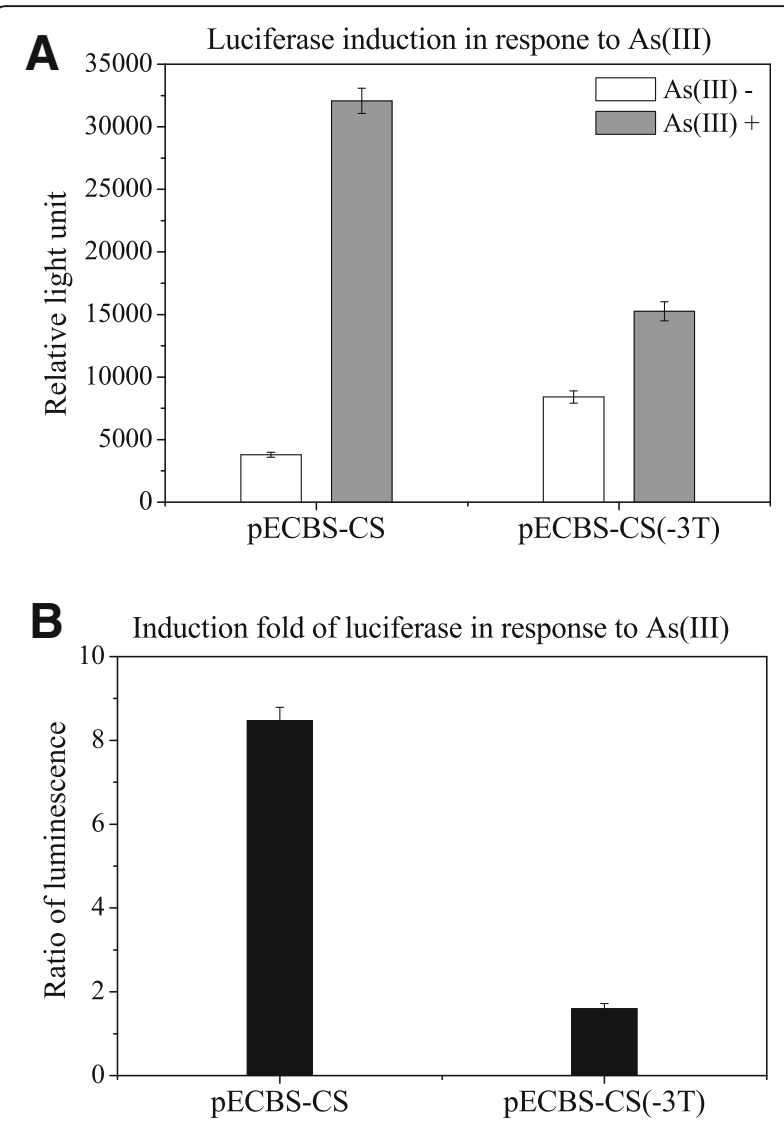

\section{ECSB-CS ECSB-CS (-3T)}

$$
\begin{array}{llllll}
1 & 2 & 3 & 1 & 2 & 3
\end{array}
$$

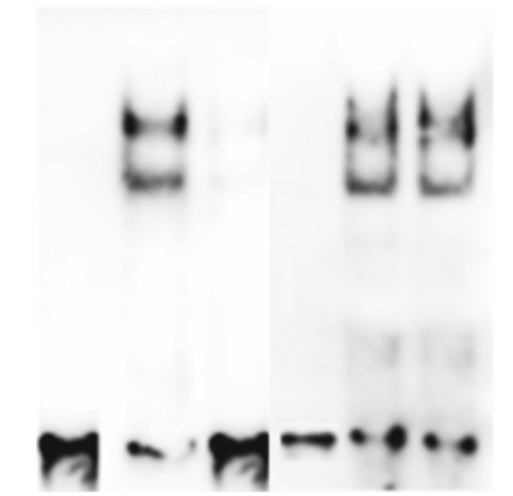

Fig. 3 Analysis of ECBS-CS and ECBS-CS(-3T) using luciferase and EMSA. A: Luciferase activities ratios of untreated to arsenic-treated cells were determined. B: Probes of ECBS-CS and ECBS-CS(-3 T) were mixed with lysates prepared from untreated and arsenictreated cells and subjected to EMSA

assay, the analysis becomes time consuming when handling a large sample size. We therefore developed a fast filter binding assay that enabled us to efficiently monitor the interaction of several probes with their binding proteins simultaneously (Fig. 4a). In this assay, probes were first mixed with lysates prepared from E. coli treated with or without arsenite, respectively. After incubation, the mixtures were loaded onto a nitrocellulose membrane (NC)-coated 96-well plate. Only protein-bound probes could stay on the plate and free probes passed through the membrane upon centrifugation. After washing, the plate was then treated with sodium dodecyl sulfate (SDS) to denature proteins, thereby releasing the probes. The released biotin-labeled probes were subjected to hybridization using a plate pre-coated with complementary sequences, and further monitored with streptavidin-HRP for luminescent detection.

To examine the feasibility of the filter assay, we employed probes ECBS-AFBS, AFBS-ECBS, ECBS-CS, CS-ECBS and ECBS-CS $(-3 \mathrm{~T})$ and mixed them with lysates prepared from arsenic-treated and mock-treated cells. The probe mixtures with lysates were first validated with EMSA before using for the filter assay. The filter assay indicated that the binding of ECBSAFBS probe with the lysate without arsenic treatment was much stronger than the lysate with arsenic treatment, and the ratio of the binding intensities of control to treated cells was about 5-fold (Fig. 4b). As expected, binding of the AFBS-ECBS probe with control and arsenic treated cell lysates was both strong and no obvious difference in binding was observed. The result with ECBS-CS probe was similar to that with the ECBS-AFBS probe and the binding ratio of control to treated cells was about 3-fold. In addition, both ECBS-CS $(-3 \mathrm{~T})$ and CS-ECBS probes displayed no difference in the binding ratio of the two different lysates (Fig. 4b). These results demonstrated that the filter assay in general was in accordance with EMSA. The only difference of the filter assay was that it could not present two distinct shifted bands like EMSA.

Next, we employed both assays to perform two-fold dilutions of lysates from both arsenic treated and control cells with the ECBS-AFBS probe. As shown in Fig. 4c, EMSA could detect the complex in 1:16 diluted lysates and filter assay in 1:64, indicating that the filter assay is 4 times more sensitive than EMSA. Therefore, the filter binding assay was able effectively to analyze the binding of several probes with target protein in a quick mode.

\section{Identification of alternations at nonconsensus base pairs crucial in protein binding using DNA filter assay}

The original E. coli ArsR binding sequence was identified as acacattcg TT AA GT CA TA TA (TG) TT TT TG AC TT A [6]. Based on the comparison with other ArsR binding sequences, we noted an extra tail of 9 base pairs at the $5^{\prime}$ end that are unlikely to contribute to arsenic-mediated induction. To reduce the cost in 


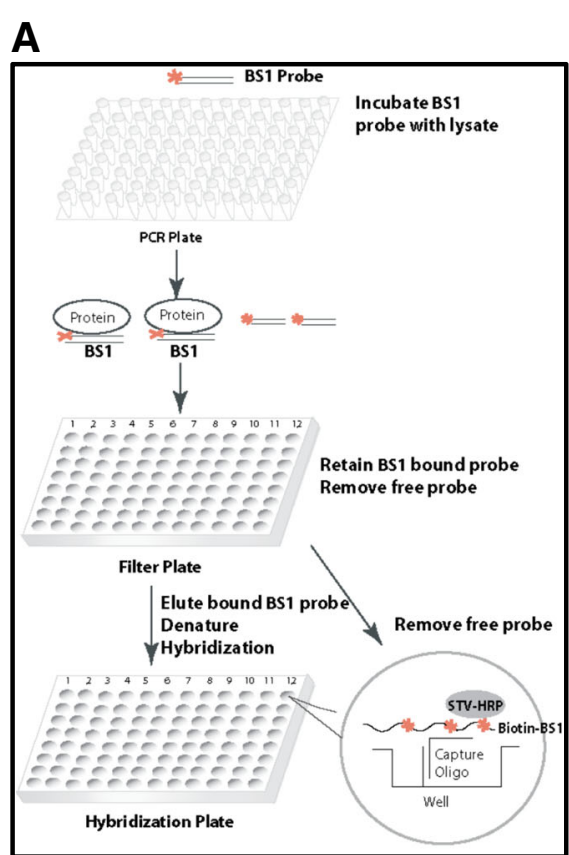

\section{B Filter assay of lysates with and without As(III)}

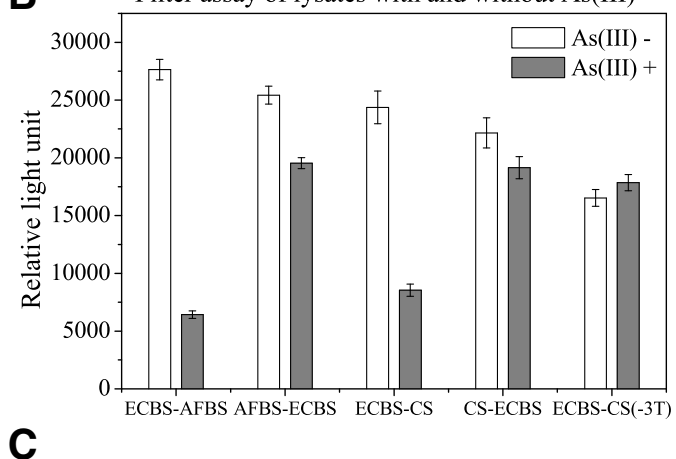

EMSA

$\begin{array}{llllll}1: 2 & 1: 4 & 1: 8 & 1: 16 & 1: 32 & 0\end{array}$

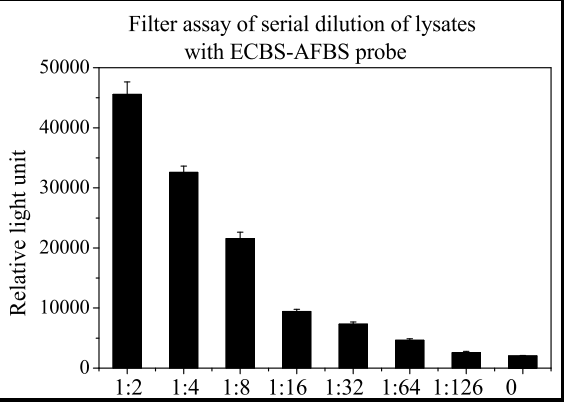

Fig. 4 Filter assay. a: Schematic diagram of the filter assay comprising 3 steps; the biotin-labeled (* indicates biotin labeled) probe BS1 (Binding Sequence) was first mixed with lysates; BS1 probe bound to the protein in the cell lysate to form protein/DNA complexes; second, the mixture loaded onto nitrocellulose (NC) membrane-based filter plate, the protein/DNA complexes retained on membrane, the free probe washed away, and the protein-bound probe eluted, denatured and hybridized on the hybridization plate; third, the hybridization and detection within one of 96 wells was illustrated in the Cycle. The capture oligos were pre-coated on the bottom of the well, the denatured biotin-labeled probe BS1 hybridized to the capture oligo, and further detected with Streptavidin Horseradish Peroxidase (STV-HRP) and measured with a luminescence plate reader. The luminescence signal directly corresponds to the binding activity. $\mathbf{b}$ : Filter assay of probes ECBS-AFBS, AFBS-ECBS, ECBS-CS, CS-ECBS and ECBS-CS(-3T) using lysates prepared from cells treated with (grey) and without (open) $10 \mu \mathrm{M}$ arsenite for $1 \mathrm{~h}$. C: Serial 2-fold dilutions of E. coli DH5a cell lysates were mixed with ECBS-AFBS probe. The mixtures were analyzed with EMSA and the filter assay respectively. The filter assay data shown are the mean values ( \pm standard deviation) obtained from three independent experiments 
oligonucleotide synthesis, we removed 5 base pairs at the $5^{\prime}$ end to make sECBS as ttcg TT AA GT CA TA TA (TG) TT TT TG AC TT A. Functional analysis using reporters with the shorter version, sECBS to replace ECBS revealed no difference (data not shown).

Among the 4 base pairs that are not conserved, two base pairs locate on each side of the inverted repeat region, TAxAxTCAAATA xx TATTTGAxTxTA, the core binding sequence was obtained by the alignment of ArsR binding sequences among $\mathrm{O} / \mathrm{P}$ sequences of arsRBC, cadCA, smtS2/S1, smtS4/S3, ziaA, czrAB, and nmtA [7]. To investigate the contribution of these nonconsensus base pairs to ArsR binding, we systematically designated different nucleotides at the position on the left and created complementary nucleotides on the right of the repeat. We constructed a series of probes with AA, TT, TA, AT CC, GG CG, GC, TC, TG, AC, AG, CT, GT, $\mathrm{CA}$, and $\mathrm{GA}$ on the left side of the repeat, as presented in Fig. 5a. Biotin-labeled probes were employed to execute the filter binding assay using cell lysate of E. coli, with or without arsenic treatment. As shown in Fig. 5b, certain probes such as sECBS-CS9m and sECBS-CS10m bound to the protein from both cell lysates, with or without arsenic treatment, were stronger than ECBSAFBS, sECBS-arsRBCBS, or sECBS-CS, whereas another probe sECBS-CS15m bound to the protein from both cell lysates, with or without arsenic treatment, was weaker than ECBS-AFBS, sECBS-arsRBCBS, or sECBSCS. Therefore, the nonconsensus base pairs could change the binding affinity to both directions. In addition to the alternations at nonconsensus base pairs, we included an alternation in the consensus base pairs to construct the probes ECBS-CS-SM as a control. With the filer binding assay, we observed that a change of 2 base pairs in the consensus sequence destroyed the difference in binding between cell lysates with and without arsenic treatment (data not shown). Moreover, we found one probe sECBS-CS12m bound the protein strongly in control cells, but weakly in arsenic-treated cells. The binding difference of this probe in control versus treated cells appeared to be the biggest among all other probes (Fig. 5c).

\section{Contribution of nonconsensus base pairs to protein binding and luciferase induction}

From the filter binding assay, we chose three probes, the strongest sECBS-CS9m in binding, the weakest sECBS-CS15m in binding, and the highest sECBSCS12m in induction, to validate the results with EMSA. As shown in Fig. 6a, the intensities of the shifted bands in EMSA were equivalent to the binding strengths in the filter binding assay, sECBS-CS9m being the strongest and sECBS-CS15m being the weakest. The difference of sECBS-CS12m shifted bands in binding intensity is the highest between control and induced cells. These results again demonstrated that changes in nonconsensus base pairs could lead to critical differences in protein binding. Importantly, our data also revealed that changes in nonconsensus base pairs could enhance arsenic-mediated removal of the bound protein, an additional functional impact after the binding is established.

To investigate the induction of few binding sequences, we used sECBS-CS12m, sECBS-CS9m, and sECBSCS15m to replace the ECBS-AFBS of pECBS-AFBS, in order to construct psECBS-CS12m, psECBS-CS9m, and psECBS-CS15m. As expected, psECBS-CS12m resulted in a better induction than the other two reporters psECBS-CS9m and psECBS-CS15m (Fig. 6b). Furthermore, we compared psECBS-CS12m with pECBS-AFBS and pECBS-CS. The transformants were treated with $10 \mu \mathrm{M}$ arsenite for 15, 30, 60 and $120 \mathrm{~min}$. As shown in Fig. 6c, arsenic-mediated induction of psECBS-CS12m was significantly better than that with either pECBSAFBS or pECBS-CS.

\section{Discussion}

Arsenic, as a naturally occurring element, is widely distributed throughout the environment. Long-term exposure to arsenic from drinking water and food can cause human diseases [16]. Prevention of further exposure to arsenic needs rapid and cost-effective onsite analytical techniques to monitor arsenic in water supplies. Bacteria-based assays are an emerging technology, in the case of arsenical contamination, to monitor arsenic-induced gene expression. Compared to the traditional capital equipment-based methods that are inappropriate for on-site detection, bacteriabased assays are robust and inexpensive for detecting arsenic in the field [17]. More significantly, they could measure arsenic bioavailability that accounts for the difference between exposure and dose [18]. The crucial component of bacteria-based assays is the reporter, comprising a promoter/operator (or an operon) and a reporter gene [19]. Ideally, a good reporter should display high sensitivity and specificity, low endogenous background, and a wide dynamic range of response [20]. In our previous study of making sensitive arsenic reporter, we constructed pLLPars9 (the same construct as pECBS-AFBS in this study) reporter and demonstrated that it is equivalent to some of the best reporters constructed to date in response to arsenic [15]. In this study, we demonstrated that the reporter psECBS-CS12m is significantly better than pLLPars9.

Metal-inducible operons contain an imperfect 12-2-12 inverted repeat, except the smt operon having two inverted repeats $\mathrm{S} 2 / \mathrm{S} 1$ and $\mathrm{S} 4 / \mathrm{S} 3$. Each repeat is 
A

\begin{tabular}{|c|c|c|c|}
\hline $\mathrm{sECBS}-\mathrm{CS} 2 \mathrm{~m}$ : & $\overline{\mathrm{T}} \overline{\mathrm{T}}$ & $\overline{\mathrm{GC}}$ & $\overline{\mathrm{A}} \overline{\mathrm{A}}$ \\
\hline sECBS-CS $5 \mathrm{~m}$ : & A A & $\mathrm{GC}$ & $\mathrm{T} \mathrm{T}$ \\
\hline sECBS-CS1m: & $\mathrm{TA}$ & $\mathrm{GC}$ & $\mathrm{T} A$ \\
\hline $\mathrm{sECBS}-\mathrm{CS} 6 \mathrm{~m}:$ & A $\mathrm{T}$ & $\mathrm{GC}$ & $\mathrm{A} T$ \\
\hline sECBS-CS11m: & $\mathrm{C} \mathrm{C}$ & $\mathrm{GC}$ & G G \\
\hline $\mathrm{sECBS}-\mathrm{CS} 16 \mathrm{~m}:$ & G G & $\mathrm{GC}$ & $\mathrm{C} \mathrm{C}$ \\
\hline sECBS-CS12m: & C G & GC & C G \\
\hline sECBS-CS15m: & G C & $\mathrm{GC}$ & G C \\
\hline sECBS-CS 3m: & $\mathrm{TC}$ & $\mathrm{GC}$ & $G A$ \\
\hline $\mathrm{sECBS}-\mathrm{CS} 4 \mathrm{~m}:$ & $\mathrm{T} \mathrm{G}$ & GC & $C A$ \\
\hline $\mathrm{sECBS}-\mathrm{CS} 7 \mathrm{~m}:$ & A C & $\mathrm{GC}$ & $\mathrm{G} T$ \\
\hline SECBS-CS 8m: & A G & $\mathrm{GC}$ & $\mathrm{C} \mathrm{T}$ \\
\hline sECBS-CS10m: & $\mathrm{C} \mathrm{T}$ & $\mathrm{GC}$ & $A G$ \\
\hline $\mathrm{sECBS}-\mathrm{CS} 14 \mathrm{~m}:$ & $\mathrm{G} T$ & $\mathrm{GC}$ & A C \\
\hline sECBS-CS 9m: & $C A$ & $\mathrm{GC}$ & $\mathrm{T} G$ \\
\hline sECBS-CS13m: & $G A$ & $\mathrm{GC}$ & $\mathrm{TC}$ \\
\hline
\end{tabular}

B The binding intensity of lysates with and without As(III)

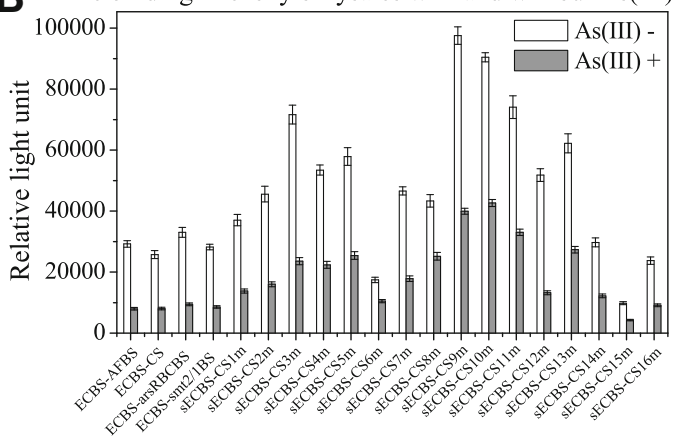

C The binding ratio of lysates with and without As(III)

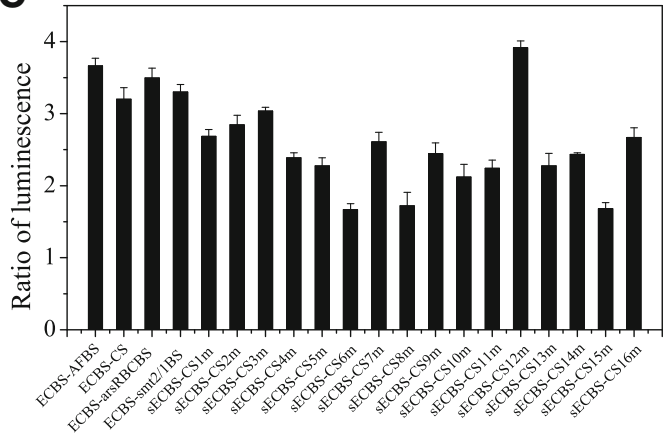

Fig. 5 Filter assay and luciferase analysis of probes with alterations at the nonconsensus base pairs. a: List of the probes with alternations at nonconsensus base pairs. $\mathbf{b}$ : The binding intensities of the probes were measured with filter assay using lysates prepared from cells treated with (grey) and without (open) $10 \mu \mathrm{M}$ arsenite for $1 \mathrm{~h}$. c: Luciferase activities ratios of untreated to treated lysates with these probes were determined. Data shown are the mean values ( \pm standard deviation) obtained from three independent experiments

occupied by an ArsR homodimer. In the previous study, we designed, such as smt operon, two binding sequences ECBS-AFBS and found that the induction of the luciferase reporter in response to the treatment of arsenic is better than either of single binding sequences [15]. We also found that the induction with these two different binding sequences is better than the two identical sequences either from EC or AF. In the present study, we uncovered that ECBS must be at the front position and the induction dramatically declined if it was replaced by other binding sequences such as smt $2 / 1$ or arsRBC. In contrast, AFBS at the second position can be replaced 

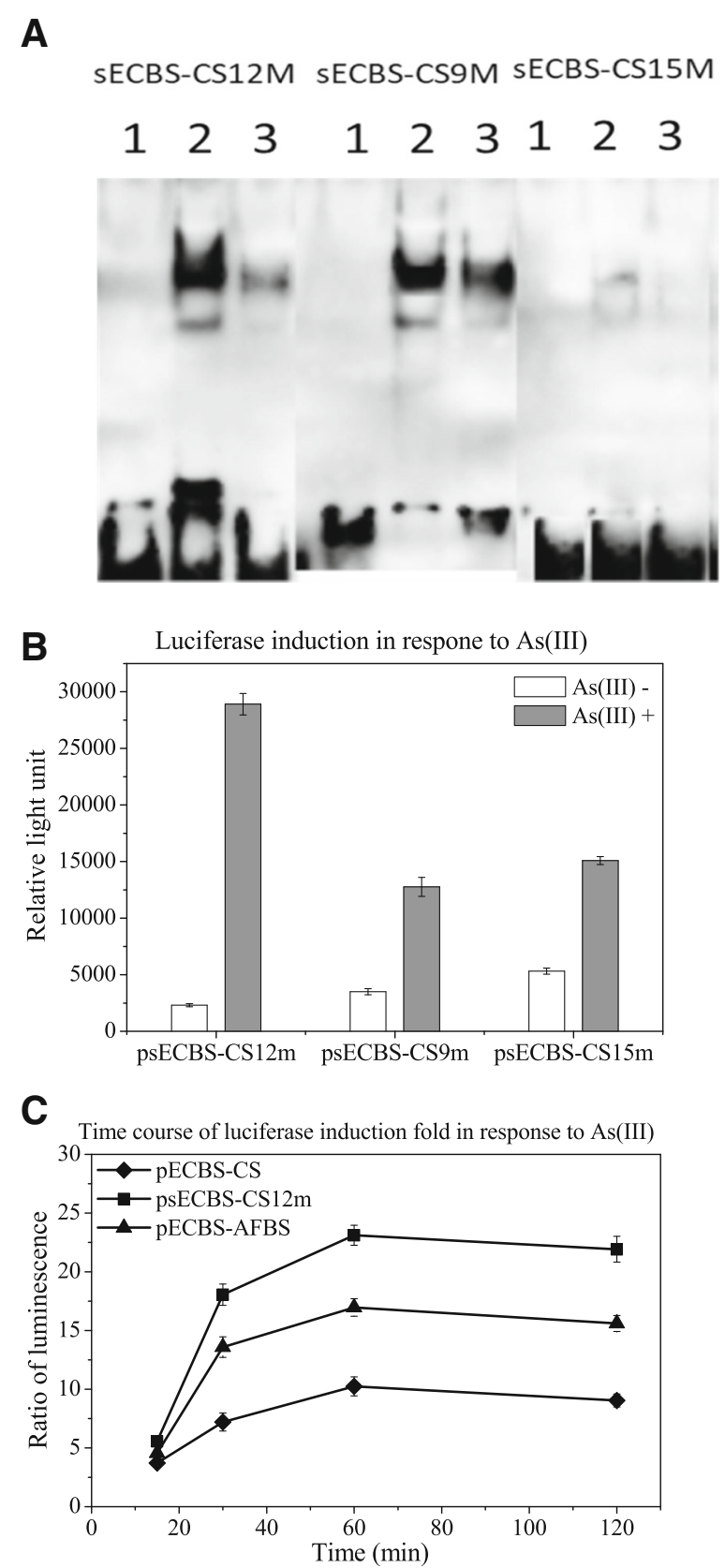

Fig. 6 EMSA and luciferase analysis of reporter constructs with the binding sequences. a: Biotin-labeled probes of sECBS-CS9m, sECBSCS12m and SECBS-CS15m were mixed with lysates prepared from untreated (open) and arsenic-treated cells (grey) and subjected to EMSA. $\mathbf{b}$ : Luciferase activities of sECBS-CS9m, sECBS-CS12m and sECBS-CS15m transformed cells pre-treated with or without $10 \mu \mathrm{M}$ arsenite for $1 \mathrm{~h}$ were measured. $\mathbf{c}$ : Time course of luciferase induction of pECBS-CS12m (square) transformed cells pre-treated with $10 \mu \mathrm{M}$ arsenite for 15, 30, 60 and 120 min were determined and compared with pECBS-AFBS (triangle) and pECBS-CS (diamond). Data shown are the mean values ( \pm standard deviation) obtained from three independent experiments by other binding sequences without affecting the induction to a significant degree. This indicated that an appropriate order of these two binding sequences is important for achieving maximal induction. As the two binding sequences bind to two dimers, the complex could be stabilized by dimer-dimer interaction [7]. Change in the order of these two binding sequences, that is AFBS-ECBS, could allow the binding of two dimers, but the order might affect arsenic interaction with the repressor protein or removal of the repressor protein from the binding sequences. Therefore, unlike ECBSAFBS, arsenic binding sites within AFBS-ECBS might be hidden due to steric structure, which prevents arsenic binding or dissociation the repressor from the binding sequence.

Protein-DNA recognition has been increasingly appreciated to be more complex than previously thought. Although the simple model of PWM has been widely used to define the DNA binding motifs of individual TFs, recent studies suggest that this model based on independent contribution of individual consensus base pairs to protein interaction is often insufficient to explain various complex regulation [8], such as the relevant dinucleotides or trinucleotides crucial to protein-DNA recognition [21-24], significant difference of low-affinity binding sites from the consensus sequence $[25,26]$, novel DNA-binding specificities of multi-protein complexes formed with a TF [27-29], and the effect of flanking sequences on the binding affinity [30]. In the present study, we employed a simpler prokaryotic ArsR regulation system to access the protein-DNA recognition. We found base pairs at nonconsensus positions within the second binding sequences such as ECBS-CS15m could result in lower binding with target protein, whereas others such as ECBS-CS9m resulted in binding with higher affinities, although both still maintained the consensus sequence. PWM was unable to explain these results. More interestingly, our study demonstrated that one of the base pairs at the nonconsensus position could also affect induction, the function beyond DNA binding. We found sECBS-CS12m, which could bind to target protein as well as ECBS-CS; however, its response to arsenic was much stronger than ECBS-CS. Their similar basal binding levels but differential induction rates suggest that arsenicmediated removal of the binding protein from the DNA binding sequence of CS12m is faster than CS. Therefore, like AFBS-ECBS, the interaction of these nonconsensus base pairs with the repressor protein could influence arsenic binding or arsenic-induced conformational change of the repressor protein, leading to differential turnover of the bound protein from the binding sequence, as exemplified by the 
observations that AFBS-ECBS was no longer sensitive to arsenic while sECBS-CS12m became more sensitive to the arsenic.

\section{Conclusions}

In the present study, we found that nonconsensus base pairs played important roles in protein-DNA binding and gene transcriptional regulation. More sensitive and accurate biosensors for arsenic detection can be developed through the design of nonconsensus base pairs. Our current findings illustrate an innovative strategy to construct better reporters, which will facilitate the development of more sensitive biosensors to monitor environmental arsenic via the induction of reporter gene expression.

\section{Methods}

\section{Plasmid construction}

Reporter constructs with different orders and sources of binding sequences were made by modifying the binding sequence of pLLPars9 [15], which was renamed as pECBS-AFBS in this study. The sense and antisense strand sequences were synthesized and annealed to generate double strand fragments with the sticky end of $\mathrm{XbaI}$ and HindIII, which were subsequently cloned into the XbaI and HindIII site of pLLPars9 [15] to replace the ECBS-AFBS to make constructs, pAFBS-ECBS, pECBS-smt2/1BS, pECBS-arsRBCBS, psmt2/1BS-ECBS, parsRBCBS-ECBS, pCS-AFBS, parsRBCBS-CS, pAFBSCS, pECBS-CS, and pECBS-CS $(-3 \mathrm{~T})$. Five base pairs at the $5^{\prime}$ end of ECBS were removed to make sECBS. Different nucleotides at the nonconsensus position of CS were designated to make CS1-16 $\mathrm{m}$. Then sECBS and CS1-16 m were subsequently cloned into the $\mathrm{XbaI}$ and HindIII site of pLLPars9 [15] to replace the ECBS-AFBS to make constructs, sECBS-CS9m, sECBS-CS12m and sECBS-CS15m.

\section{Luciferase assay}

E. coli $\mathrm{DH} 5 \alpha$ competent cells were transformed with the recombinant plasmids constructed in this study. Single colonies were picked and inoculated in $2 \mathrm{~mL}$ LuriaBertani (LB) media supplied with $25 \mu \mathrm{g} / \mathrm{mL}$ chloramphenicol for $12-16 \mathrm{~h}$ at $37^{\circ} \mathrm{C}$ with vigorous shaking. The overnight culture was 1:50 diluted in a $1.5 \mathrm{~mL}$ microcentrifuge tube with pre-warm and fresh-prepared $2 \mathrm{~mL} \mathrm{LB}$ media supplied with chloramphenicol. The diluted cells were cultured for additional $3 \mathrm{~h}$ at $37{ }^{\circ} \mathrm{C}$ until the optical density (OD) reached 0.5 . Cells were treated with or without $10 \mu \mathrm{M}$ sodium arsenite [As (III)] for $60 \mathrm{~min}$ at $37^{\circ} \mathrm{C}$. The cell samples were sonicated to lyse the cells, and the protein concentration was measured with Bradford Protein Assay (Bio-Rad, Cat\#5000201) to confirm the equal protein concentration among the treated and untreated cell samples. Twenty $\mu \mathrm{L}$ of induced sample was taken and mixed $50 \mu \mathrm{L}$ luciferase substrate, and the luciferase activities were measured on the luminescence plate reader (Veritas).

\section{Preparation of cell lysates}

One $\mathrm{mL}$ of cell culture with or without sodium arsenite was centrifuged at 10,000 g for $1 \mathrm{~min}$ and the pellet was resuspended in $300 \mu \mathrm{L}$ of lysis buffer $(10 \mathrm{mM}$ Tris- $\mathrm{HCl}$, $\mathrm{pH}$ 8.0, $0.1 \mathrm{M} \mathrm{NaCl}, 1 \mathrm{mM}$ ethylene diamine tetraacetic acid (EDTA), and $0.1 \%[\mathrm{w} / \mathrm{v}]$ polyethylene glycol octylphenol ether (TRITON X-100)). Senve point five $\mu \mathrm{L}$ of a freshly prepared lysozyme solution $(10 \mathrm{mg} / \mathrm{mL}$ in $10 \mathrm{mM}$ Tris- $\mathrm{HCl}, \mathrm{pH} 8.0$, final concentration is $0.25 \mathrm{mg} / \mathrm{mL}$ ) was added and mixed well by tapping the tube gently, and the lysis mixture was incubated for $10-20 \mathrm{~min}$ at room temperature. After centrifugation, the supernatant was used for electrophoretic mobility shift assay (EMSA) or Filter assay.

\section{EMSA}

One to $3 \mu \mathrm{g}$ cell lysate was mixed with $2 \mu \mathrm{L}$ of $5 \times$ binding buffer and $1 \mu \mathrm{L}$ polyd(I-C) and incubated on ice for $5 \mathrm{~min}$. One $\mu \mathrm{L}$ of biotin-labeled probe was added to the mixture and incubated at $22^{\circ} \mathrm{C}$ for $30 \mathrm{~min}$. Each reaction mixture was separated using a $6.5 \%$ non-denaturing polyacrylamide gel at $100 \mathrm{~V}$ at $4{ }^{\circ} \mathrm{C}$ in $0.5 \times$ Tris-borateEDTA (TBE) for about 50 to $60 \mathrm{~min}$. After the gel was transferred onto an $\mathrm{NC}$ membrane and blocked by adding $15 \mathrm{~mL}$ of blocking buffer for $20 \mathrm{~min}$ at room temperature, the biotin-labeled probe on the blot was then detected with streptavidin-HRP and chemiluminescent substrates (enhanced chemiluminescence by luminol, Pierce). The image was acquired using an imager.

\section{Filter assay method}

In this assay, $2 \mu \mathrm{L}$ cell lysate $(2-10 \mu \mathrm{g})$ was mixed with $10 \mu \mathrm{L} \mathrm{2 \times}$ Binding Buffer Mix (40 mM 4-(2-hydroxyethyl)-1-piperazineethanesulfonic acid (HEPES), $\mathrm{pH}$ 7.6, $20 \mathrm{mM}$ ammonia sulfate, $2 \mathrm{mM}$ dithiothreitol (DTT), $20 \mathrm{mM} \mathrm{KCl}$, and $0.4 \%$ Tween-20), $1 \mu \mathrm{L}$ biotin-

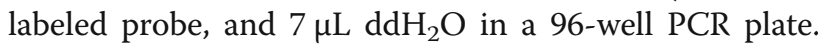
After incubation at room temperature for $30 \mathrm{~min}$, the reaction mix was loaded onto to a prewashed filter assay plate and incubated on ice for $20 \mathrm{~min}$, following which it was centrifuged at $600 \mathrm{~g}$ for $2 \mathrm{~min}$. The flow-through was discarded and the plate was washed for 4 times with filter wash buffer $(100 \mathrm{mM}$ Tris- $\mathrm{HCl}, \mathrm{pH} 7.6,2.5 \mathrm{mM}$ EDTA, and $0.1 \%$ Tween-20). The bound probe was eluted with Elution buffer (0.5\% SDS). The eluted probe was heated at $95^{\circ} \mathrm{C}$ for $3 \mathrm{~min}$ before hybridization. Hybridization was carried out by adding the eluted DNA probe to a plate pre-coated with corresponding DNA and incubating at $42{ }^{\circ} \mathrm{C}$ overnight. After wash, the bound 
probe was eluted from the filter and collected for quantitative analysis through DNA plate hybridization. The captured DNA probe was further detected with streptavidin-HRP and the signals were read by a luminescence plate reader (Beckman Coulter, LD-400), and reported as relative light units (RLUs). Induction fold was the ratio of luminescence of arsenic-treated cells to that of arsenic-untreated cells.

\begin{abstract}
Abbreviations
AFBS: binding sequences from A. ferrooxidans; ArsR: arsenic repressor; arsRBC: R773 arsR operon; arsRBCBS: binding sequence from arsRBC CS: consensus sequence of arsRBC and cadCA; DTT: dithiothreitol; ECBS: binding sequences from E. coli; EDTA: ethylene diamine tetraacetic acid; EMSA: electrophoretic mobility shift assay; HEPES: 4-(2-hydroxyethyl)-1 piperazineethanesulfonic acid; LB: Luria-Bertani media; NC: nitrocellulose membrane; O/P: operator/promoter; OD: optical density; PWM: position weight matrix; RLUs: relative light units; SDS: sodium dodecyl sulfate; smt2/ 1BS: binding sequence from Synechococcus smt2/1; TBE: Tris-borate-EDTA

TF: transcription factor; TRITON X-100: polyethylene glycol octylphenol ether
\end{abstract}

\section{Acknowledgements}

Thank Professors Yinghua Cen and Xiangdong Fu for reading the manuscript.

\section{Authors' contributions}

YW, MX, GS, JG, and XL developed the protocol. XC, XJ, CT, and JY performed the experiments. $X C, X J, M X$, and $X L$ analyzed the data and wrote the manuscript. $X C$ and $X J$ contributed equally to this work. All authors read and approved the final manuscript.

\section{Funding}

This work was supported by the High-level Leading Talent Introduction Program of GDAS (2016GDASRC-0208) and the Science and Technology Planning Project of Guangzhou City (201707020021) to XL, the National Natural Science Foundation of China (21677042) and the Natural Science Foundation of Guangdong Province (2018B0303110010) to XC, and the National Natural Science Foundation of China $(91851202,51678163)$ to MX.

\section{Availability of data and materials}

All data generated or analysed during this study are included in this published article.

\section{Ethics approval and consent to participate}

Not applicable.

\section{Consent for publication}

Not applicable.

\section{Competing interests}

The authors declare that they have no competing interests.

\section{Author details}

${ }^{1}$ Guangdong Provincial Key Laboratory of Microbial Culture Collection and Application, Guangdong Institute of Microbiology, Guangzhou, China. ${ }^{2}$ State Key Laboratory of Applied Microbiology Southern China, Guangzhou, China. ${ }^{3}$ Science and Technology Library of Guangdong Province, Guangdong Institute of Science and Technology Information and Development Strategy, Guangzhou, China. ${ }^{4}$ Signosis Inc., 1700 Wyatt Drive, suite10-12, Santa Clara, CA, USA.

Received: 12 March 2019 Accepted: 27 May 2019

Published online: 06 June 2019

\section{References}

1. Vaquerizas JM, Kummerfeld SK, Teichmann SA, Luscombe NM. A census of human transcription factors: function, expression and evolution. Nat Rev Genet. 2009;10(4):252-63.
2. Albert FW, Kruglyak $\mathrm{L}$. The role of regulatory variation in complex traits and disease. Nat Rev Genet. 2015;16(4):197-212.

3. Stormo GD, Schneider TD, Gold L, Ehrenfeucht A. Use of the 'perceptron' algorithm to distinguish translational initiation sites in E. coli. Nucleic Acids Res. 1982;10(9):2997-3011.

4. Stormo GD. Modeling the specificity of protein-DNA interactions. Quant Biol. 2013;1(2):115-30

5. Inukai S, Kock KH, Bulyk ML. Transcription factor-DNA binding: beyond binding site motifs. Curr Opin Genet Dev. 2017;43:110-9.

6. Xu C, Shi W, Rosen BP. The chromosome arsR gene of Escherichia coli encodes a trans-acting metalloregulatory protein. J Biol Chem. 1996;271(5): 2427-32.

7. Busenlehner LS, Pennella MA, Giedroc DP. The SmtB/ArsR family of metalloregulatory transcriptional repressors: structural insights into prokaryotic metal resistance. FEMS Microbiol Rev. 2003:27(2-3):131-43.

8. Siggers T, Gordan R. Protein-DNA binding: complexities and multi-protein codes. Nucleic Acids Res. 2014:42(4):2099-111.

9. San Francisco MJ, Hope CL, Owolabi JB, Tisa LS, Rosen BP. Identification of the metalloregulatory element of the plasmid-encoded arsenical resistance operon. Nucleic Acids Res. 1990;18(3):619-24.

10. Wu J, Rosen BP. Metalloregulated expression of the ars operon. J Biol Chem 1993;268(1):52-8.

11. Shi W, Wu J, Rosen BP. Identification of a putative metal binding site in a new family of metalloregulatory proteins. J Biol Chem. 1994;269(31): 19826-9.

12. Qin J, Fu HL, Ye J, Bencze KZ, Stemmler TL, Rawlings DE, Rosen BP. Convergent evolution of a new arsenic binding site in the ArsR/SmtB family of metalloregulators. J Biol Chem. 2007;282(47):34346-55.

13. Butcher BG, Rawlings DE. The divergent chromosomal ars operon of Acidithiobacillus ferrooxidans is regulated by an atypical ArsR protein. Microbiology. 2002;148(Pt 12:3983-92

14. Hödar C, Moreno P, Genova A, Latorre M, Reyes-Jara A, Maass A, González M, Cambiazo V. Genome wide identification of Acidithiobacillus ferrooxidans (ATCC 23270) transcription factors and comparative analysis of ArsR and MerR metal regulators. Biometals. 2012;25(1):75-93.

15. Fang $Y$, Zhu $C$, Chen X, Wang Y, Xu M, Sun G, Guo J, Yoo J, Tie C, Jiang X, Li $X$. Copy number of ArsR reporter plasmid determines its arsenite response and metal specificity. Appl Microbiol Biotechnol. 2018;102(13):5753-61.

16. Jomova K, Jenisova Z, Feszterova M, Baros S, Liska J, Hudecova D, Rhodes CJ, Valko M. Arsenic: toxicity, oxidative stress and human disease. J Appl Toxicol. 2011;31(2):95-107.

17. Chen J, Rosen BP. Biosensors for inorganic and organic arsenicals. Biosensors (Basel). 2014:4(4):494-512.

18. Soangra R, Majumder B, Roy P. Whole cell arsenic biosensor - a cheap technology for bioavailable arsenic (as) determination. Eur J Adv Eng Technol. 2015;2:52-61.

19. Close DM, Ripp S, Sayler GS. Reporter proteins in whole-cell optical bioreporter detection systems, biosensor integrations, and biosensing applications. Sensors. 2009:9(11):9147-74.

20. Kaur H, Kumar R, Babu JN, Mittal S. Advances in arsenic biosensor development - a comprehensive review. Biosens Bioelectron. 2015 63(15):533-45.

21. Gershenzon NI, Stormo GD, loshikhes IP. Computational technique for improvement of the position-weight matrices for the DNA/protein binding sites. Nucleic Acids Res. 2005;33(7):2290-301.

22. Zhao Y, Ruan S, Pandey M, Stormo GD. Improved models for transcription factor binding site identification using nonindependent interactions. Genetics. 2012:191(3):781-90.

23. Weirauch MT, Cote A, Norel R, Annala M, Zhao Y, Riley TR, Saez-Rodriguez J, Cokelaer T, Vedenko A, Talukder S. Evaluation of methods for modeling transcription factor sequence specificity. Nat Biotechnol. 2013;31(2):126-34.

24. Mathelier A, Wasserman WW. The next generation of transcription factor binding site prediction. PLoS Comput Biol. 2013;9(9):e1003214.

25. Jaeger SA, Chan ET, Berger MF, Stottmann R, Hughes TR, Bulyk ML. Conservation and regulatory associations of a wide affinity range of mouse transcription factor binding sites. Genomics. 2010;95(4):185-95.

26. Tanay A. Extensive low-affinity transcriptional interactions in the yeast genome. Genome Res. 2006:16(8):962-72.

27. Perkins AS, Fishel R, Jenkins NA, Copeland NG. Evi-1, a murine zinc finger proto-oncogene, encodes a sequence-specific DNA-binding protein. Mol Cell Biol. 1991;11(5):2665-74 
28. Delwel R, Funabiki T, Kreider BL, Morishita K, Ihle JN. Four of the seven zinc fingers of the Evi-1 myeloid-transforming gene are required for sequence-specific binding to GA(C/T)AAGA(T/C)AAGATAA. Mol Cell Biol. 1993;13(7):4291-300

29. Funabiki T, Kreider BL, Ihle JN. The carboxyl domain of zinc fingers of the Evi-1 myeloid transforming gene binds a consensus sequence of GAAG ATGAG. Oncogene. 1994;9(6):1575-81.

30. Gordan R, Shen N, Dror I, Zhou T, Horton J, Rohs R, Bulyk ML. Genomic regions flanking $\mathrm{E}$-box binding sites influence DNA binding specificity of bHLH transcription factors through DNA shape. Cell Rep. 2013;3(4): 1093-104.

\section{Publisher's Note}

Springer Nature remains neutral with regard to jurisdictional claims in published maps and institutional affiliations.

Ready to submit your research? Choose BMC and benefit from:

- fast, convenient online submission

- thorough peer review by experienced researchers in your field

- rapid publication on acceptance

- support for research data, including large and complex data types

- gold Open Access which fosters wider collaboration and increased citations

- maximum visibility for your research: over $100 \mathrm{M}$ website views per year

At BMC, research is always in progress.

Learn more biomedcentral.com/submissions 